\title{
Image-guided video assisted thoracoscopic surgery (iVATS) and the future of surgical management
}

\author{
Desiree A. Steimer^, Abby White \\ Division of Thoracic Surgery, Brigham and Women's Hospital and Harvard Medical School, Boston, Massachusetts, USA. \\ Contributions: (I) Conception and design: Both authors; (II) Administrative support: Both authors; (III) Provision of study materials or patients: \\ Both authors; (IV) Collection and assembly of data: Both authors; (V) Data analysis and interpretation: Both authors; (VI) Manuscript writing: Both \\ authors; (VII) Final approval of manuscript: Both authors. \\ Correspondence to: Abby White, DO. Division of Thoracic Surgery, Brigham and Women's Hospital, 75 Francis Street, Boston, MA 02115, USA. \\ Email: Awhite12@bwh.harvard.edu.
}

\begin{abstract}
The increased utilization of computed tomography for diagnostic imaging has led to an influx of incidentally discovered pulmonary nodules. Both thoracic surgeons and radiologists are burdened with risk stratifying these lesions for observation versus biopsy or resection. The diagnostic accuracy of percutaneous biopsy is dependent on the lesion's size, density, and anatomic location. Nodules that are not amenable to percutaneous biopsy are often referred to surgeons for excisional biopsy. Certain characteristics make some lesions more difficult to resect than others. Small $(<1 \mathrm{~cm})$, partially solid, pure ground glass, and central lesions can be difficult to palpate intraoperatively. These lesions can be challenging to resect with thoracoscopic and robotic assisted approaches due to diminished tactile feedback and smaller incisions. Over the past 30 years, techniques have been developed to assist surgeons in locating these lesions with the use of hook wires, fiducials, dyes, and radiolabeled isotopes. These markers are placed preoperatively but are prone to migration or diffusion. In this chapter a new localization and resection technique, image-guided video assisted thoracoscopic surgery (iVATS) is reviewed. iVATS is an attractive option for both patients and surgeons since the localization procedure and parenchymal resection are performed under a single anesthetic. A detailed description of the iVATS technique, success rate, and common complications are reviewed. For pulmonary nodules that are centered within a defined bronchovascular segment, upfront segmentectomy is a reasonable option for obtaining pathologic diagnosis. Since segmentectomy relies on surgeon knowledge and experience with segmental anatomy, non-palpable lesions can be easily treated with this technique. Pearls and pitfalls of the most common segmental resections are addressed. Despite the refinement of localization techniques, small pulmonary nodules continue to present a diagnostic challenge. Ongoing innovation of new clinical tools has generated promising options for the future. In this chapter, we will review some of the tools undergoing development including computer generated algorithms for nodule risk assessment, liquid biopsy, three-dimensional printing, and superselective segmentectomy. Some technologies are focused on creating non-invasive methods to determine if a nodule is benign or malignant, while others seek to improve localization techniques. All of the described tools will need further validation but will potentially streamline the diagnosis and treatment of pulmonary nodules for future clinical practice.
\end{abstract}

Keywords: Image-guided video assisted thoracoscopic surgery (iVATS); segmentectomy; pulmonary nodule; localization techniques

Received: 20 May 2020; Accepted: 26 March 2021; Published: 25 November 2022.

doi: $10.21037 /$ ccts-20-102

View this article at: http://dx.doi.org/10.21037/ccts-20-102

$\wedge$ ORCID: 0000-0003-0945-3455. 


\section{Introduction}

The increased utilization and improved image quality of computed tomography (CT) scanners has influenced the detection rate of small $(\leq 1 \mathrm{~cm})$ pulmonary nodules, making this a common referral for thoracic surgeons. A thoughtful approach is required to delicately balance avoiding unnecessary procedures while not missing early-stage malignancies. Unfortunately, positron emission tomography (PET) often adds little diagnostic value in evaluating these lesions that are just at or below the threshold for PET sensitivity (1).

Pure ground glass opacities (GGO), partially solid, and solid nodules less than $1 \mathrm{~cm}$ can be managed with close observation, percutaneous biopsy, navigational biopsy, or excisional biopsy, depending on clinical factors and associated imaging findings (2-4). Percutaneous or navigational biopsy may fail to obtain a diagnosis due to inadequate sampling of the target lesion (2-9). The accuracy of biopsy diminishes with smaller $(<1 \mathrm{~cm})$ targets and pure ground glass lesions (2). Some nodules, especially central ones, may not be amenable to percutaneous or navigational biopsy due to their anatomic location $(3,6,10)$. For instance, lesions behind a rib or under the scapula cannot be accessed percutaneously and peripheral lesions may not be accessible via navigational bronchoscopy. In such patients, surgical excision remains the definitive approach for both diagnosis and treatment $(4,5,11,12)$.

While a peripheral, solid pulmonary nodule may be readily palpable, lesions that are partially solid, centrally located, or less than $1 \mathrm{~cm}$ in size can be difficult to locate intraoperatively $(2-4,6,10,12,13)$. For these lesions, it is more feasible to identify them with localization techniques prior to resection $(2,3,6,8,9,14,15)$. An alternative approach, for lesions centered within bronchovascular segments, is to perform anatomic segmentectomy (16). Both of these surgical strategies can be accomplished safely with minimally invasive techniques and are parenchymal-sparing while providing ample tissue architecture for diagnosis and molecular profiling. Preservation of lung parenchyma becomes important when definitively treating patients with multiple lung nodules or with limited pulmonary reserve $(2,16)$. This chapter will review current surgical strategies as well as potential future tools to assist in the management of small $(\leq 1 \mathrm{~cm})$ pulmonary nodules.

\section{Localization techniques}

Locating small pulmonary nodules intraoperatively can be challenging, especially with minimally invasive techniques that utilize smaller incisions and limit tactile feedback $(3,10,12,17-20)$. Localization techniques were developed to assist thoracic surgeons in identifying these indistinct, non-palpable lesions $(4,7,8,17-20)$. The technique initially described a hook wire being placed into the target lesion under fluoroscopic or CT guidance and then immediately followed by video assisted thoracoscopic surgery (VATS) resection $(19,20)$. Intraoperatively, a core of lung parenchyma surrounding the wire was resected using a laser (argon or neodymium:yttrium aluminum garnet) (19). Over the years, this technique has evolved and now includes a wider variety of markers to facilitate resection (gold fiducials, methylene blue dye, radiolabeled isotopes) $(5,8,21)$. The reported overall success of this two-step approach locating the target lesion ranges from $90 \%$ to $93 \%(4,13,20)$. The major limitation of preoperative localization is the delay between marker placement and operative incision, leaving opportunity for wire migration, dye spillage, or dye fading $(4-8,12,13,21)$.

These shortcomings prompted the creation of localization techniques that could be performed intraoperatively such as ultrasound (21), placement of fiducials (7) or wires (22), and injection of methylene blue $(7,17,23)$ or indocyanine green dye $(7,18,22)$. Markers can be placed alone or in combination with one another $(7,22)$; they are commonly delivered via navigational bronchoscopy $(7,18,23)$, electromagnetic transthoracic injection (17) or in hybrid operating rooms with CT capabilities $(2,3,11,22,24)$. At our institution, many of these intraoperative techniques have been performed to facilitate lesion localization with the newest being imageguided video assisted thoracoscopic surgery (iVATS).

\section{iVATS-an overview of technique and outcomes}

To address the challenge of resecting non-palpable lung lesions, iVATS was developed through collaborations between radiology, anesthesiology, and thoracic surgery specialties at our institution as well as others internationally $(2,3,10,11,13,15,24)$. iVATS is performed in a hybrid operating room with CT capabilities; this technique performs both localization and surgical resection under a single anesthetic $(2,3,10,11,13,15,24)$.

\section{Positioning}

After double-lumen endotracheal tube placement, the patient is positioned according to the localization plan. Some programs perform marker localization in the supine 


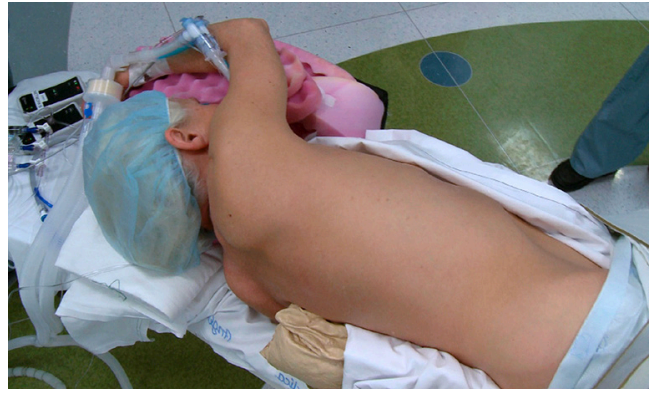

Figure 1 Standard lateral decubitus positioning for an iVATS case. Arms should be positioned cephalad to avoid collisions with $\mathrm{C}$-arm during procedure. iVATS, image-guided video assisted thoracoscopic surgery.

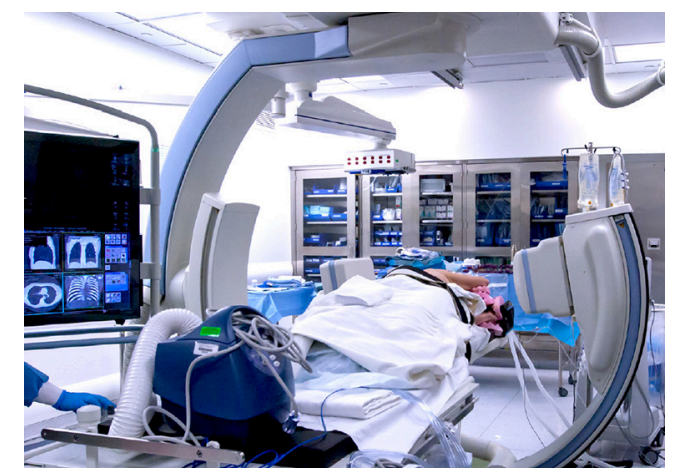

Figure 2 Patient undergoes initial C-arm computed tomography scan in our hybrid operating room. Images obtained map a trajectory for guided marker placement.

or prone position when necessary $(11,15,24)$. Lateral decubitus position is preferred for marker placement at our institution as this eliminates the need for re-positioning for the VATS portion of the operation $(2,3)$. For hybrid operating rooms equipped with a C-arm CT scanner, it is important to have the arms positioned cephalad to avoid collisions during scanning (Figure 1). The ventilator circuit, intravenous and arterial lines need extension tubing to ensure adequate length during scans (2,3). A test scan is advised to confirm that there are no positioning issues prior to prepping the patient. Team members from anesthesia, radiology, and surgery must all be satisfied with positioning prior to proceeding $(2,3)$.

\section{Procedure review}

Preoperative CT images are utilized to compile a three- dimensional (3D) model for multidisciplinary discussion to optimize marker placement $(2,3)$. After the patient is positioned on the hybrid table, an initial CT scan is performed with an inspiratory hold of 5-8 seconds (Figure 2) $(2,3)$. Once the target lesion is confirmed, iGuide needle guidance software (Siemens Healthcare AG, Forchieim, Germany) assists in trajectory planning for markers $(2,3)$. The needle pathways are integrated into the $\mathrm{C}$-arm imaging system which projects laser crosshairs on the patient's skin (Figure 3) and provides guidance markers for fluoroscopy to direct needle angles and improve success of T-bar placement (Kimberly-Clark, Roswell, GA, USA) (2,3). Under sterile conditions with the lung inflated, T-bars are placed by the surgeon. Markers are placed just immediately deep to the target without crossing into the lesion itself. The T-bar should exit the lung parenchyma relatively close to the target to minimize the resection required to remove the lesion and T-bar within the specimen (Figure 4). The thoracic radiologist is heavily involved in optimizing targeting and execution of marker placement; it is a true multidisciplinary approach.

The operative lung is deflated after marker placement and the patient is prepped and draped for VATS. Three incisions are used to allow resection of the target lung parenchyma and T-bars (Figure 5). Once removed from the patient via an endo-bag, the specimen is physically and radiographically inspected to ensure complete resection of the T-bar(s) and the nodule (Figure 6) (2,3). Frozen section analysis provides pathologic diagnosis and margin status; additional parenchymal resection is performed if indicated based on margin distance or diagnosis $(2,3)$.

\section{Localization success rate}

Our institution has two published iVATS series with a composite experience of 75 patients; there was an $87 \%$ success rate of nodule localization in the first series and $97 \%$ in the second $(2,3)$. Reasons for localization failure included equipment malfunction $(1.3 \%)$ and dislodged T-bar (2.8\%) (2,3). All target lesions were resected despite marker migration (2). There were no reported deaths at 30 or 90 days $(2,3)$.

Similar techniques for iVATS have been reported using dye injection for peripheral lesions and hook wire localization for deeper ones $(10,11,15,24)$. The overall procedural success rate is cited between $90-100 \%$ at these institutions $(10,11,15,24)$. The most common reason for localization failure at other programs was unsuccessful 


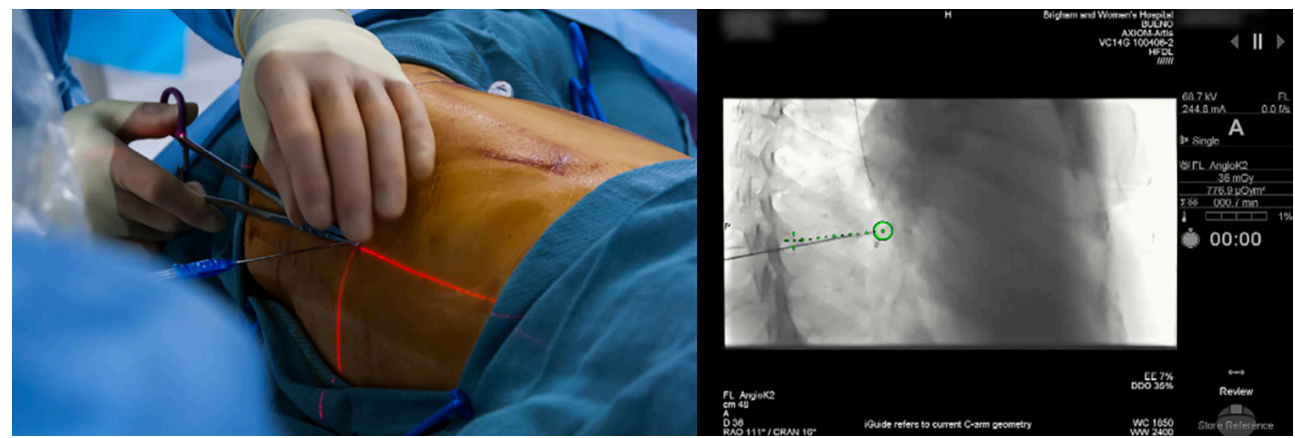

Figure 3 Laser crosshair from C-arm imaging system facilitates percutaneous T-bar placement for lesion localization (left). Real time fluoroscopic guidance optimizes needle trajectory and T-bar placement within desired target using iGuide software (right).

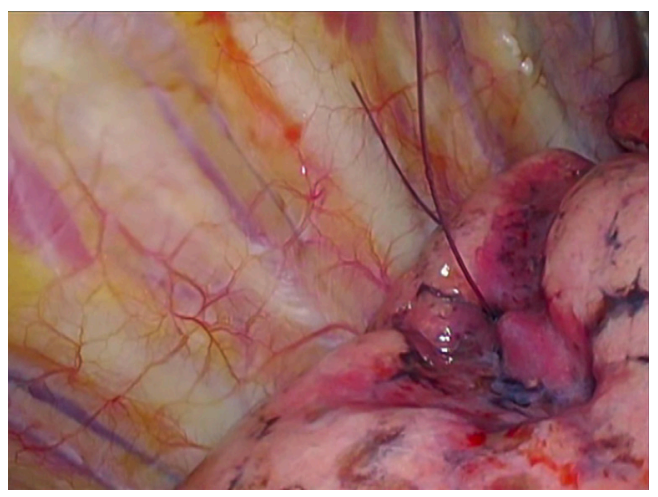

Figure 4 Intraoperative image demonstrating tail of T-bar exiting lung parenchyma in the superior segment of the lower lobe and easily identifies the location of target lesion.

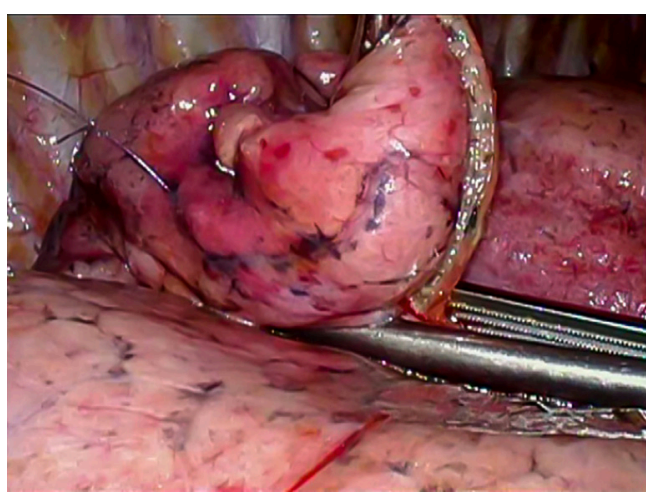

Figure 5 A limited parenchymal resection is performed thoracoscopically. Care is taken during stapling to include both the target lesion and localization marker within a single specimen.

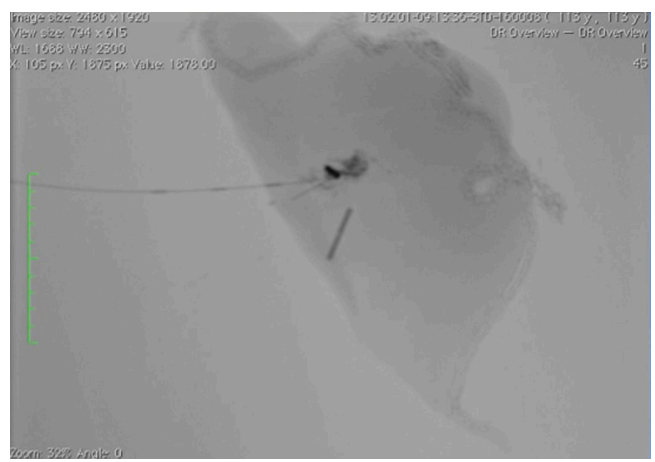

Figure 6 After the specimen is removed from the patient, it is inspected radiographically with computed tomography scan to ensure the desired lesion and T-bars have been resected.

targeting due to pneumothorax $(2.4 \%)(11,15,24)$. Other etiologies of overall procedural failure were equipment malfunction $(2.2 \%)$, wire dislodgement $(4.3 \%)$, and dye spillage $(3.3 \%)(11,15,24)$. There were no perioperative deaths reported (11).

\section{Complications}

Introduction of dye or wires into the lung parenchyma can result in pneumothorax or hemothorax, even with careful image guidance (2-4,6,10-13,24). At our institution, localization related pneumothorax rate was $6.8 \%(2,3)$, however, other iVATS centers have reported rates ranging from $2.1 \%$ to $47 \%(10,11,13,15,24)$. Hemothorax from marker placement has occurred less frequently and has been 
reported in $0.7-2.9 \%$ of cases $(13,24)$. These complications were not clinically significant and were promptly addressed at time of VATS $(2,3,10,11,13,15,24)$. In contrast to iVATS, preoperative wire localization has more associated complications: pneumothorax $58 \%(4,6,12,13)$, hemothorax $16.6 \%(6,12,13)$, intercostal hematoma $7.2 \%$ (4), hemoptysis $2.6 \%$ (12), retained wire 1\% (4), and systemic air embolism $0.2 \%$ (12). These complications can occur at any time after the procedure and may occur when the patient is not yet under care of the surgeon, thus delaying any necessary intervention. Pneumothorax was the most common complication to require intervention prior to operative resection and was due to worsening clinical status from hypoxia or dyspnea (12).

While retained foreign body is a concern with hook wires, T-bars, and fiducials, all T-bars were recovered in our first iVATS series, including those that became dislodged from the target lesion (2). In the second series, however, there were three T-bars that were retained (3.1\%) (3). These were embedded within the diaphragm, liver and chest wall and could not be safely removed (3). Other iVATS centers do not specifically address if any localization markers were retained in their cases $(10,11,13,15,24)$.

\section{Advantages and limitations of technique}

Wire localization of small pulmonary nodules has been utilized in thoracic surgery for nearly 30 years (19-20). Conventional preoperative wire placement followed by VATS resection has significant variability in time between localization and surgical procedures depending on institutional workflow $(6,12,13)$. Some centers report mean wait times of 3.6 hours (13) while others are as long as 27 hours (12). These delays can not only result in wire displacement or loss $(9.5-22 \%)$, but leave patients vulnerable to untreated complications such as pneumothorax, hemothorax and hemoptysis $(2,4,10,13,20,24)$. In contrast, complications occurring from marker placement can be managed quickly in iVATS cases since patients are already anesthetized and in the operating suite $(2,3,13,24)$. A prospective study was conducted and directly compared preoperative localization to iVATS technique (13). The time between marker placement and surgical incision (time at risk) was significantly longer for patients undergoing preoperative localization (215 minutes preoperative vs. 13 minutes iVATS, $\mathrm{P}<0.001)$ (13). The overall success of targeting was similar between the approaches ( $91 \%$ vs. $93 \%, \mathrm{P}=0.3$ ), but pneumothorax rate was significantly higher in the preoperative localization cohort compared to the iVATS group $(46.7 \%$ vs. $2.9 \%, \mathrm{P}<0.001)(13)$.

Most centers perform all wire placements in the lateral decubitus position $(2,3,10,13)$. Eliminating the additional step of re-positioning decreases procedure time and provides less opportunity for marker migration $(2,3,13,15)$. For iVATS, the average cited rate of wire displacement is $3.3 \%(2,3,10,11,15,24)$. In contrast, preoperative localization with hook wires has a migration rate ranging between $9.5 \%$ and $22 \%$ depending on the institution $(4,20)$. Loss of localization marker, especially for deep lesions, can result in aborted procedures, enlarged incisions, conversion to thoracotomy to palpate lesion, or lobectomy to ensure the lesion is resected $(4,6,9,11,17,18,21)$.

CT guidance for placement of localization markers offers several advantages. First, targeting CT images can re-evaluate the lesion and determine if there is any change in size and/or density. Second, the post-localization scan provides information about the relationship between your marker and target lesion that can be valuable when performing the resection $(2,8)$. Other delivery methods for localization such as navigational bronchoscopy and electromagnetic transthoracic techniques cannot provide feedback on marker location after it is placed (8).

Although there are many benefits to iVATS, the technique has some limitations. Two key resources are required to execute these cases effectively; a dedicated multidisciplinary team and a hybrid operating room. Hybrid operating rooms are equipped with advanced imaging technology such as C-arm CT scanners. Unfortunately, not all institutions have access to hybrid operative suites and this is the main limitation of this technique. Another crucial component of this procedure is the skill and experience level of the multidisciplinary team performing the iVATS cases. A dedicated group of providers from anesthesia, radiology, and thoracic surgery is essential for the success of these procedures. Lastly, the technique is relatively new and has only been evaluated in small patient series from single institution experiences $(2,3,10,11,13,15,24)$. Although it is difficult to draw conclusions from these limited cohorts, it is clear that the technique is safe and feasible since multiple institutions have independently created iVATS protocols $(2,3,10,11,13,15,24)$.

iVATS has improved upon the conventional two-step approach for localization of small pulmonary nodules. It has good localization success rate and very few procedure related complications $(2,3,10,11,13,15,24)$. Patients may prefer this approach because care is streamlined and 
patient centered; some may report less anxiety and physical discomfort since marker placement is performed under general anesthesia (24). Additionally, the technique allows thoracic surgeons to intervene on smaller nodules and GGOs that previously were managed with observation due to challenges surrounding resection $(2,3,11)$. Resection of these lesions when they are early stage, node negative lung cancers will eliminate the need for adjuvant therapies (chemotherapy and/or radiation) and hopefully improve patient disease-free survival $(2,3,25)$.

\section{Segmentectomy}

Anatomic segmentectomy is an alternative strategy for management of small pulmonary lesions. Ideal lesions for segmentectomy are peripheral, less than $2 \mathrm{~cm}$ in size, and well centered within the target segment in order to achieve appropriate margins (26-31). Caution should be taken for lesions that cross intersegmental planes, as margins are unlikely to be adequate (26). Careful review of preoperative CT images with thoracic radiologists is advised to be able to identify lesions within specific pulmonary segments. Contrast enhanced images (CT arteriograms) and 3D reconstructions can be helpful to surgeons for preoperative planning but are not universally available.

In contrast to wedge resections, segmentectomy requires dissection and division of bronchovascular structures which improves mobilization for a deeper parenchymal margin and allows thorough evaluation of draining lymph nodes $(28,30)$. Lesions that are confirmed malignancies on frozen section do not obligate additional resection as long as margins are adequate. It is recommended to obtain at least $2 \mathrm{~cm}$ margins to decrease risk of disease recurrence (26-27,32). As for all anatomic resections for lung cancer, adequate lymph node sampling is essential for appropriate staging (25). Though current literature is beginning to shed light on this topic, conversion to a lobectomy is advised if $\mathrm{N} 1$ positive lymph nodes are encountered.

VATS segmentectomy requires a firm foundational knowledge of segmental planes and vascular anatomy $(16,23,29,31)$. All segmentectomies are not equal in their level of difficulty; the superior segment is the least technically challenging while individual basilar segments are more complex operations $(23,26)$. Typically, a VATS segmentectomy can be performed with three incisions: an inferior port for the camera, a posterior port for retraction, and an anterior utility incision (16). Initial inspection of the hemithorax evaluates for resectability of the lesion and evidence of more advanced disease, such as nodal metastasis or pleural carcinomatosis. For most anatomic lung resections, dissection begins with the segmental vein followed by the segmental pulmonary artery (PA). Division of vascular structures is performed after both vein and artery branches are dissected free (16). Lastly, the segmental bronchus is isolated and divided; anatomy can be confirmed prior to division by intraoperative bronchoscopy or gentle lung re-inflation to ensure the correct bronchus is divided (16).

Similar to VATS lobectomy, there are unique anatomic challenges to each segmentectomy with some common pitfalls for the various operations (16). Although superior segmentectomy is one of the more straightforward operations (26), PA anatomy is highly variable and there can be up to three superior segmental PA branches. All superior segmental branches and the posterior ascending branch to the upper lobe should be identified prior to division of the posterior fissure. Dissection of the superior segmental bronchus can lead the surgeon toward the superior branches of the inferior pulmonary vein, and care must be taken to avoid avulsing these small branches.

A composite basilar segmentectomy preserves the superior segment of the lower lobe. After the superior segmental and middle lobe PA branches have been identified, dividing the posterior and anterior fissures can gain mobility of the basilar segment and accommodate improved angles for stapling the basilar PA (16).

For a lingular-sparing lobectomy (apical trisegmentectomy), dissection of the upper division vein is first. Care must be taken to preserve venous branches to the lingula, which can be easily traced to that segment (Figure 7). Next, dissection of anterior branches of the PA is performed. This is followed by dissection in the fissure or along the posterior hilar reflection in order to expose the posterior ascending PA branches. Due to the variable PA anatomy of the left upper lobe, confirmation of the presence of a lingular artery is advised prior to the division of arterial branches. This avoids congestion which can make it difficult to create intersegmental planes during the parenchymal division. During dissection of the proximal PA branches and level 5 lymph node, careful attention must be paid to avoid injuring the recurrent laryngeal and vagus nerves (16).

Lingulectomy is functionally similar to a middle lobectomy, but the proximity of the lingular bronchus to the ongoing PA can make this dissection challenging. The lingular artery should be isolated prior to division of the lingular vein branch. During dissection of the lingular 


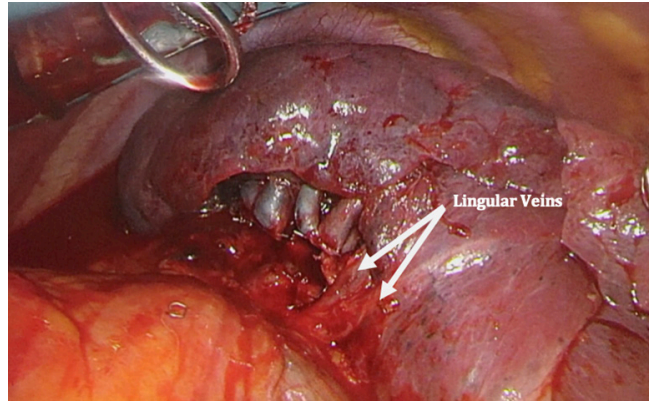

Figure 7 Two lingular veins are spared during left upper lobe trisegmentectomy. It is crucial to identify a lingular artery in the fissure and ensure adequate blood supply to the remaining lingula.

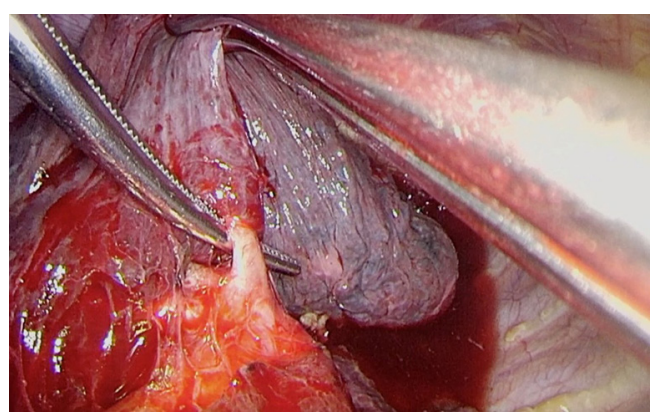

Figure 8 The left upper lobe posterior artery is dissected for posterior segmentectomy. The bronchus to this segment can be found immediately posterior and parallel to the artery. Two lingular branches are seen coursing anteromedially.

bronchus, care is necessary to sweep tissue off the ongoing PA inferiorly. Dissecting lymph nodes at the vascular branch points helps to delineate the anatomy further for dissection and allows analysis of "sump" lymph nodes.

Posterior segmentectomy can be performed in either upper lobe and typically starts with dissection of the PA in the posterior fissure (Figure 8). The bronchus is often found just posterior and running parallel to the artery. It is rare to encounter a specific large venous branch to the posterior segment.

Anterior segmentectomy of the right upper lobe can be performed by initial dissection that mirrors a right upper lobectomy. The superior most branch of the superior pulmonary vein is divided and the anterior branch of the PA dissected and divided (Figure 9).

For an apical segmentectomy, the bronchus is often located posteriorly with the arterial branch immediately behind. Care must be taken not to "overshoot" when

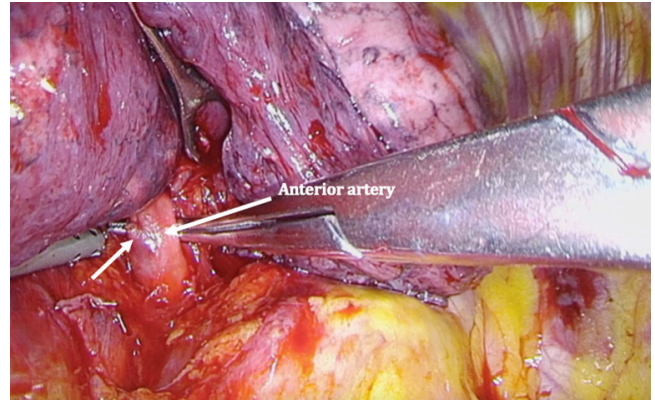

Figure 9 The vein to the anterior segment of the right upper lobe has been divided with an endovascular stapler (foreground). The pulmonary artery is dissected next. A small branch of the artery has been divided with the Harmonic scalpel to avoid avulsion during dissection.

passing instruments behind the bronchus so as to avoid avulsing the segmental PA at this level.

\section{Emerging technologies and the future of surgical management}

Small pulmonary nodules and GGOs will continue to present challenges both in diagnosis and management for thoracic surgeons now and in the future. Tools that enhance the ability to identify which nodules require intervention and which can be safely monitored will be exceedingly useful and are areas of active innovation. Some developing tools include surgical techniques such as superselective segmentectomy, 3D printing for localization planning, computer generated algorithms, and non-invasive tools such as liquid biopsy.

Superselective segmentectomy is a diagnostic tool that was developed to address small, deep pulmonary nodules and GGOs that are not amenable to wedge resection (33). This technique preserves more lung parenchyma than traditional segmentectomy but is challenging to perform due to anatomic variability of the distal PA branches (33). Preoperative 3D CT angiography provides a detailed map of the arterial anatomy and its relationship to the target lesion (33). The described technique is performed through a posterolateral thoracotomy and intersegmental plane is developed bluntly; no staplers are used for parenchymal division (33). There were no perioperative complications or deaths in their series (33). This prospective trial of 30 patients determined that superselective segmentectomy is a feasible option to establish tissue diagnosis (33), but whether this is an acceptable alternative to segmentectomy or 
lobectomy for treatment of lung cancer remains unclear. An advantage of segmentectomy is that it is both a diagnostic and therapeutic procedure for patients with indeterminate pulmonary nodules that are subsequently diagnosed as stage I lung cancer $(28,30)$. For superselective segmentectomy patients diagnosed with lung cancer, they required a second thoracotomy and completion lobectomy as treatment unless they were medically unfit to tolerate additional pulmonary resection (33). The main limitation of the technique is the technical complexity; it takes an average of 4 hours to perform this operation (33). A clear benefit to superselective segmentectomy is the amount of lung parenchyma spared during resection, which is appealing for patients with multiple pulmonary nodules. In its current state, it is not widely applicable to most practices and there are alternative surgical options that can be performed minimally invasively while this technique continues to be refined.

There are many methods for nodule localization, each with associated advantages and limitations. An ideal localization technique would be easy-to-use, accurate, have no additional radiation exposure to patient and staff and be performed intraoperatively to minimize complications. One institution has developed a $3 \mathrm{D}$ printed targeting tool to assist in placement of hook wires (14). 3D printed templates have the advantage of being customizable to individual patients based on established CT images (14); image resolution of 1 to $1.5 \mathrm{~mm}$ thickness is required for optimal printing $(14,23)$. Currently this template is being validated and used in combination with preoperative CT guidance for hook wire placement (14). With refinement of the technique it could be used as an intraoperative localization tool and obviate the need for additional CT use. Currently the template has only been used on patients with low body mass index (median 22.7), so it is not generalizable to all populations in its current state of development (14).

One of the major challenges of small pulmonary nodules is determining if they are benign or malignant. Although there are certain radiographic features that are suggestive of malignancy, radiologists and surgeons are not perfect at differentiating between the two. Resections of "suspicious" pulmonary lesions with either preoperative or intraoperative localization techniques have an $8 \%$ to $50 \%$ reported rate of benign diagnoses (granuloma, hamartoma, pneumonia) on final pathology $(3,6-9,11,18,24)$. To address this issue, Maldonado et al. created a program called "computeraided nodule assessment and risk yield (CANARY)" and compared this tool to independent thoracic radiologist interpretations of high-resolution CT chest images (34).
All patients within the training and validation sets had surgically resected adenocarcinoma spectrum lesions that were less than $3 \mathrm{~cm}$ in size (34). CANARY was highly sensitive and specific in determining if a lesion was aggressive (invasive adenocarcinoma) or indolent (in situ or minimally invasive adenocarcinoma) (34). Interestingly, the two senior radiologists had a lower accuracy rate than CANARY in correctly categorizing the lesions (34). There was also significant interobserver variability between the two radiologists; in $~ 20 \%$ of the cases the two physicians disagreed on the aggressiveness of the tumor (34). Further refinement of the tool is underway with the aim to be able to risk stratify lesions as low, intermediate or high risk. This may potentially reduce the number of unnecessary resections for benign pulmonary lesions.

Another promising tool to help differentiate benign and malignant lesions in development is liquid biopsy. This technology relies on analyzing nuclear material from tumor cells within the patient's blood $(35,36)$. A tumor must contain a minimum of 50 million cells for adequate levels of DNA to be detected by this method (35). Application of circulating DNA assays are currently limited in lung cancer patients and only approved by the Food and Drug Administration for assessment of EGFR mutations in patients with biopsy proven non-small cell lung cancer $(35,36)$. Assays that analyze circulating tumor cells are approved for use in patients with advanced breast and colorectal cancers; these tests are useful in prognosis since high numbers of circulating tumor cells often correlate with poor clinical outcomes (35). This test is not yet approved for patients with lung cancer, but studies suggest this assay may have higher accuracy in patients with small cell compared to non-small cell lung cancers (35). Currently, liquid biopsy techniques are useful in determining the burden of disease and response to treatment for patients with known lung cancer, but they are not validated to independently make a lung cancer diagnosis $(35,36)$. This approach is clearly advantageous to patients that do not desire additional percutaneous biopsies, have a technically challenging lesion to re-biopsy, and patients with multiple medical comorbidities making them poor biopsy candidates $(35,36)$. In the future, liquid biopsy may be able to provide the diagnosis of new lung cancer and/or recurrent lung cancer and potentially help direct treatment.

\section{Discussion}

Small nodules and GGOs remain a clinical challenge for 
thoracic surgeons despite ongoing innovations to simplify localization and resection. At our institution, many surgeons perform VATS segmentectomy to manage these suspicious lesions when they are within a defined anatomic lung segment. While segmentectomy is a good option for management of these lesions, they can be challenging to perform and anatomic location of the target must be favorable. For patients that are not segmentectomy candidates, iVATS is particularly useful. iVATS carries the advantage of expedient localization and resection. After resection, CT imaging confirms that the target is removed as well as the inserted markers. This technique spares lung parenchyma for patients with benign etiologies of nodules and can be useful in re-operative patients with difficult adhesions that distort normal anatomy. Fortunately, both approaches can be performed minimally invasively and are parenchymal conserving, allowing for treatment of any additional nodules that may arise.

There are many described techniques for nodule localization; choosing the right one for an individual practice requires assessment of hospital resources and surgical experience. New techniques and non-operative innovations will continue to be developed and expand the available options within the thoracic surgeon's armamentarium.

\section{Acknowledgments}

We would like to thank Dr. Raphael Bueno and Dr. Ritu Gill for their helpful insights on the iVATS technique and for allowing us to reproduce photographs of the operation. We would like to acknowledge Emily Polhemus for her assistance obtaining intraoperative images for the segmentectomy figures.

Funding: None.

\section{Footnote}

Provenance and Peer Review: This article was commissioned by the Guest Editors (Scott Swanson, Daniel Dolan) for the series "How to Evaluate, Diagnose and Treat Small Lung Nodules" published in Current Challenges in Thoracic Surgery. The article has undergone external peer review.

Peer Review File: Available at https://ccts.amegroups.com/ article/view/10.21037/ccts-20-102/prf

Conflicts of Interest: Both authors have completed the
ICMJE uniform disclosure form (available at https://ccts. amegroups.com/article/view/10.21037/ccts-20-102/coif). The series "How to Evaluate, Diagnose and Treat Small Lung Nodules" was commissioned by the editorial office without any funding or sponsorship. The authors have no other conflicts of interest to declare.

Ethical Statement: The authors are accountable for all aspects of the work in ensuring that questions related to the accuracy or integrity of any part of the work are appropriately investigated and resolved. Informed consent was obtained from patients prior to photography of iVATS cases previously; images used in this manuscript are reproductions of copyrighted photographs. Intraoperative segmentectomy photographs have no discernable features to compromise patient anonymity, but consent was obtained from the patients to film their surgeries.

Open Access Statement: This is an Open Access article distributed in accordance with the Creative Commons Attribution-NonCommercial-NoDerivs 4.0 International License (CC BY-NC-ND 4.0), which permits the noncommercial replication and distribution of the article with the strict proviso that no changes or edits are made and the original work is properly cited (including links to both the formal publication through the relevant DOI and the license). See: https://creativecommons.org/licenses/by-nc-nd/4.0/.

\section{References}

1. Kernstine KH, Grannis FW and Rotter AJ. Is There a Role for PET in the Evaluation of Subcentimeter Pulmonary Nodules? Semin Thorac Cardiovasc Surg 2005;17:110-4.

2. Gill RR, Zheng Y, Barlow JS, et al. Image-guided Video Assisted Thoracoscopic Surgery (iVATS) - Phase I-II Clinical Trial. J Surg Oncol 2015;112:18-25.

3. Gill RR, Barlow J, Jaklitsch MT, et al. Image-guided videoassisted thoracoscopic resection (iVATS): Translation to clinical practice-real-world experience. J Surg Oncol 2020;121:1225-32.

4. Thaete FL, Peterson MS, Plunkett MB, et al. Computed Tomography-Guided Wire Localization of Pulmonary Lesions Before Thoracoscopic Resection: Results in 101 Cases. J Thorac Imaging 1999;14:90-8.

5. Nardini M, Bilancia R, Paul I, et al. Technetium and methylene blue guided pulmonary nodules resection: preliminary British experience. J Thorac Dis 
2018;10:1015-21.

6. Seo JM, Lee HY, Kim HK, et al. Factors determining successful computed tomography-guided localization of lung nodules. J Thorac Cardiovasc Surg 2012;143:809-14.

7. Abbas A, Kadakia S, Ambur V, et al. Intraoperative electromagnetic navigational bronchoscopic localization of small, deep or subsolid pulmonary nodules. J Thorac Cardiovasc Surg 2017;153:1581-90.

8. Sancheti MS, Lee R, Ahmed SU, et al. Percutaneous Fiducial Localization for Thoracoscopic Wedge Resection of Small Pulmonary Nodules. Ann Thorac Surg 2014;97:1914-8; discussion 1919.

9. Suzuki K, Nagai K, Yoshida J, et al. Video-Assisted Thoracoscopic Surgery for Small Indeterminate Pulmonary Nodules: indications for preoperative marking. Chest 1999;115:563-8.

10. Yu PS, Chu CM, Lau RWH, et al. Video-assisted thoracic surgery for tiny pulmonary nodules with real-time image guidance in the hybrid theatre: the initial experience. J Thorac Dis 2018;10:2933-9.

11. Chao YK, Wen CT, Fang HY, et al. A single-center experience of 100 image-guided video-assisted thoracoscopic surgery procedures. J Thorac Dis 2018;10:S1624-30.

12. Ichinose J, Kohno T, Fujimori S, et al. Efficacy and Complications of Computed Tomography-Guided Hook Wire Localization. Ann Thorac Surg 2013;96:1203-8.

13. Chao YK, Pan KT, Wen CT, et al. A comparison of efficacy and safety of preoperative versus intraoperative computed tomography-guided thoracoscopic lung resection. J Thorac Cardiovasc Surg 2018;156:19741983.e1.

14. Zhang L, Li M, Li Z, et al. Three-dimensional printing of navigational template in localization of pulmonary nodule: A pilot study. J Thorac Cardiovasc Surg 2017;154:21132119.e7.

15. Hsieh MJ, Wen CT, Fang HY, et al. Learning curve of image-guided video-assisted thoracoscopic surgery for small pulmonary nodules: A prospective analysis of 30 initial patients. J Thorac Cardiovasc Surg 2018;155:18251832.e1.

16. White A, Swanson SJ. Video-assisted thoracic surgery (VATS) segmentectomy: state of the art. Minerva Chir 2016;71:61-6.

17. Long J, Petrov R, Haithcock, B, et al. Electromagnetic Transthoracic Nodule Localization for Minimally Invasive Pulmonary Resection. Ann Thorac Surg 2019;108:1528-34.
18. Geraci TC, Ferrari-Light D, Kent A, et al. Technique, Outcomes with Navigational Bronchoscopy Using Indocyanine Green for Robotic Segmentectomy. Ann Thorac Surg 2019;108:363-9.

19. Mack MJ, Gordon MJ, Postma TW, et al. Percutaneous Localization of Pulmonary Nodules for Thoracoscopic Lung Resection. Ann Thorac Surg 1992;53:1123-4.

20. Plunkett MB, Peterson MS, Landreneau RJ, et al. Peripheral Pulmonary Nodules: Preoperative Percutaneous Needle Localization with CT Guidance. Radiology 1992;185:274-6.

21. Mack, MJ, Shennib H, Landreneau RJ, et al. Techniques for localization of pulmonary nodules for thoracoscopic resection. J Thorac Cardiovasc Surg 1993;106:550-3.

22. Ujiie H, Kato $\mathrm{T}, \mathrm{Hu} \mathrm{H}$, et al. A novel minimally invasive near-infrared thoracoscopic localization technique of small pulmonary nodules: A phase I feasibility trial. J Thorac Cardiovasc Surg 2017;154:702-11.

23. Abdelsattar ZM and Blackmon SH. Using novel technology to augment complex video-assisted thoracoscopic single basilar segmentectomy. J Thorac Dis 2018;10:S1168-78.

24. Leow OQY, Chao YK. Individualized Strategies for Intraoperative Localization of Non-palpable Pulmonary Nodules in a Hybrid Operating Room. Front Surg 2019;6:32.

25. National Comprehensive Cancer Network guidelines for Non-Small Cell Lung Cancer. (accessed April 26, 2020). Available online: https://www.nccn.org/

26. Swanson SJ. Video-Assisted Thoracic Surgery Segmentectomy: The Future of Surgery for Lung Cancer. Ann Thorac Surg 2010;89:S2096-7.

27. Schuchert MJ, Pettiford BL, Keeley S, et al. Anatomic Segmentectomy in the Treatment of Stage I Non-Small Cell Lung Cancer. Ann Thorac Surg 2007;84:926-32; discussion 932-3.

28. Okada M, Koike T, Higashiyama M, et al. Radical sublobar resection for small-sized non-small cell lung cancer: a multicenter study. J Thorac Cardiovasc Surg 2006;132:769-75.

29. Shapiro M, Weiser TS, Wisnivesky JP, et al. Thoracoscopic segmentectomy compares favorably with thoracoscopic lobectomy for patients with small stage I lung cancer. J Thorac Cardiovasc Surg 2009;137:1388-93.

30. Schuchert MJ, Kilic, A, Pennathur A, et al. Oncologic Outcomes After Surgical Resection of Subcentimeter NonSmall Cell Lung Cancer. Ann Thorac Surg 2011;91:16817; discussion 1687-8. 
31. Bilgi Z, Swanson SJ. Current indications and outcomes for thoracoscopic segmentectomy for early stage lung cancer. J Thorac Dis 2019;11:S1662-9.

32. Mohiuddin K, Haneuse S, Sofer T, et al. Relationship between margin distance and local recurrence among patients undergoing wedge resection for small $(<2 \mathrm{~cm})$ non-small cell lung cancer. J Thorac Cardiovasc Surg 2014;147:1169-75.

33. Nakamoto K, Omori K, Nezu K, et al. Superselective segmentectomy for deep and small pulmonary nodules under the guidance of three-dimensional reconstructed computed tomographic angiography. Ann Thorac Surg 2010;89:877-83.

34. Maldonado F, Boland JM, Raghunath S, et al. Noninvasive
Characterization of the Histopathologic Features of Pulmonary Nodules of the Lung Adenocarcinoma Spectrum using Computer-Aided Nodule Assessment and Risk Yield (CANARY) - A Pilot Study. J Thorac Oncol 2013;8:452-60.

35. Sholl LM, Aisner DL, Allen TC, et al. Liquid Biopsy in Lung Cancer: A Perspective From Members of the Pulmonary Pathology Society. Arch Pathol Lab Med 2016;140:825-9.

36. Sacher AG, Komatsubara KM and Oxnard GR. Application of Plasma Genotyping Technologies in NonSmall Cell Lung Cancer: A Practical Review. J Thorac Oncol 2017;12:1344-56. doi: $10.21037 /$ ccts-20-102

Cite this article as: Steimer DA, White A. Image-guided video assisted thoracoscopic surgery (iVATS) and the future of surgical management. Curr Chall Thorac Surg 2022;4:37. 